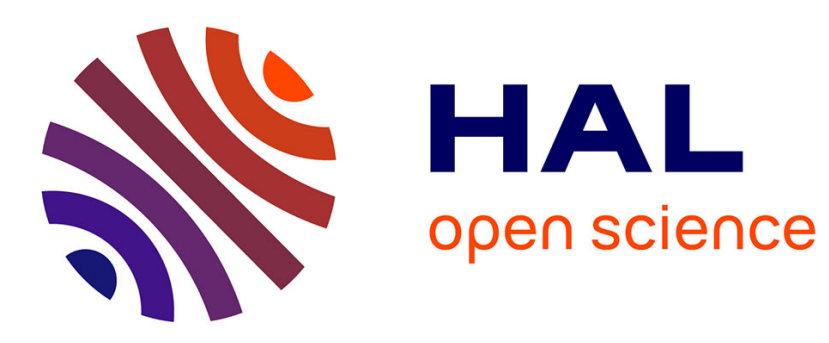

\title{
Glass Transition Accelerates the Spreading of Polar Solvents on a Soluble Polymer
}

Julien Dupas, Emilie Verneuil, Maxime van Landeghem, Bruno Bresson, Laurent Forny, Marco Ramaioli, Francois Lequeux, Laurence Talini

\section{- To cite this version:}

Julien Dupas, Emilie Verneuil, Maxime van Landeghem, Bruno Bresson, Laurent Forny, et al.. Glass Transition Accelerates the Spreading of Polar Solvents on a Soluble Polymer. Physical Review Letters, 2014, 112 (18), pp.188302 10.1103/PhysRevLett.112.188302 . hal-01406913

\section{HAL Id: hal-01406913 \\ https://hal.science/hal-01406913}

Submitted on 1 Dec 2016

HAL is a multi-disciplinary open access archive for the deposit and dissemination of scientific research documents, whether they are published or not. The documents may come from teaching and research institutions in France or abroad, or from public or private research centers.
L'archive ouverte pluridisciplinaire HAL, est destinée au dépôt et à la diffusion de documents scientifiques de niveau recherche, publiés ou non, émanant des établissements d'enseignement et de recherche français ou étrangers, des laboratoires publics ou privés. 


\title{
Glass transition accelerates the spreading of polar solvents on a soluble polymer
}

\author{
Julien Dupas, ${ }^{1}$ Emilie Verneuil, ${ }^{1}$ Maxime Van Landeghem, ${ }^{1}$ Bruno Bresson, ${ }^{1}$ \\ Laurent Forny, ${ }^{2}$ Marco Ramaioli, ${ }^{2}$ Francois Lequeux, ${ }^{1}$ and Laurence Talini ${ }^{1}$ \\ ${ }^{1}$ CNRS/UPMC/ESPCI ParisTech, UMR 7615, Laboratoire SIMM, 10 rue Vauquelin, 75231 Paris, France \\ ${ }^{2}$ Nestle Research Center, route du Jorat 57, 1000 Lausanne 26, Switzerland
}

(Dated: April 25, 2014)

\begin{abstract}
We study the wetting of polymer layers by polar solvents. As previously observed, when a droplet of solvent spreads, both its contact angle and velocity decrease with time as a result of solvent transfers from the droplet to the substrate. We show that, when the polymer is initially glassy, the angle decreases steeply for a given value of the velocity, $U_{g}$. We demonstrate that those variations result from a plasticization, i.e. a glass transition, undergone by the polymer layer during spreading, owing to the increase of its solvent content. By analyzing previous predictions on the wetting of rigid and soft viscoelastic substrates, we relate $U_{g}$ to the viscosity of the polymer gel close to glass transition. Finally, we derive an analytical prediction for $U_{g}$, based on existing predictions for the water transfer from the droplet to the substrate. Using polar solvents of different natures, we show that the experimental data compares well to the predicted expression for $U_{g}$.
\end{abstract}

Poor dissolution of powders leading to lump formation is a common experience in industrial processes as well as in everyday life, however its causes remain poorly understood. It is generally admitted that wetting of powders by a liquid is the first step for dispersing powders in that liquid, and that it often constitutes the rate controlling process, rather than the following steps of imbibition and final dispersion [1]. In the past decades, a large experimental and theoretical effort has been devoted to the understanding of the wetting of non soluble substrates $[2,3]$, including polymeric substrates [4], but wetting by their solvent of soluble materials has been investigated only recently [5-7]. A straightforward experiment consists in depositing a droplet of solvent on a soluble substrate, and in simultaneously measuring the wetting angle and contact line velocity of the spreading droplet. Complex wetting behaviors have been reported in that simple geometry; in particular, a counter-intuitive effect is observed with polar solvents: large dynamic wetting angles can be measured although the substrate is soluble $[5,6,8]$. That behavior has been attributed to the exhibition of the material apolar groups to the interface with air [8]. Since the affinity of the solvent for the substrate increases as the substrate gets solvated, the wetting dynamics is controlled by the solvent content in the substrate [9]. Furthermore, analyses of the different solvent transfer processes from the droplet to the substrate have shown the major role of transport through the vapor phase $[5,8,10]$. In the course of a spreading experiment, the solvent content in the substrate ahead the contact line increases, owing mostly to condensation of the solvent evaporated from the droplet; as a consequence, the wetting angle strongly decreases together with the contact line velocity during spreading $[6,7,10]$. Solvent sorption is therefore a crucial parameter for the wetting of soluble materials, and the non-linearities in the sorption isotherm exhibited by polymeric substrates and their solvents can result in complex behaviors [7]. The solvoscopy, i.e. uptake of solvent versus vapor concentration increase, plays a critical role.

Many polymers involved in dissolution processes are in a glassy state at room temperature since amorphous forms generally have faster dissolution rates compared to their crystalline counterparts [11]. Nevertheless, when their solvent volume fraction increases, they can undergo a plasticization, i.e. a glass transition in solvent content, and reach a melt state. Glass transition is expected to strongly influence the wetting dynamics since it induces large variations of the solvent diffusion coefficient and solvent uptake in the polymer, as well as drastic changes in the mechanical behaviour (see Fig. 1). Within a small range of solvent content, the polymer evolves from a rigid solid, poorly solvoscopic and in which the solvent has a small diffusion coefficient, to a soft viscoelastic material, highly solvoscopic with a large solvent diffusion coefficient. The influence of the mechanical properties of the substrate on wetting dynamics has been established in the case of non soluble substrates: when soft and viscoelastic, the substrate is locally deformed at the contact line and the viscoelastic dissipation in the substrate may be larger than the viscous dissipation in the liquid [12-14] thus substantially impeding wetting. In the present work, we investigate the case of a substrate undergoing glass transition during the course of spreading, the transition resulting from the transfer of solvent from the spreading droplet to the substrate at room temperature. We show that the wetting angle of a polar solvent on an initially glassy polymer film abruptly decreases during spreading. We demonstrate how that effect results from glass transition, and we develop a model to quantitatively predict the velocity at which glass transition plays a role. We confirm the validity of our predictions with experiments performed with polar solvents of different volatilities. 

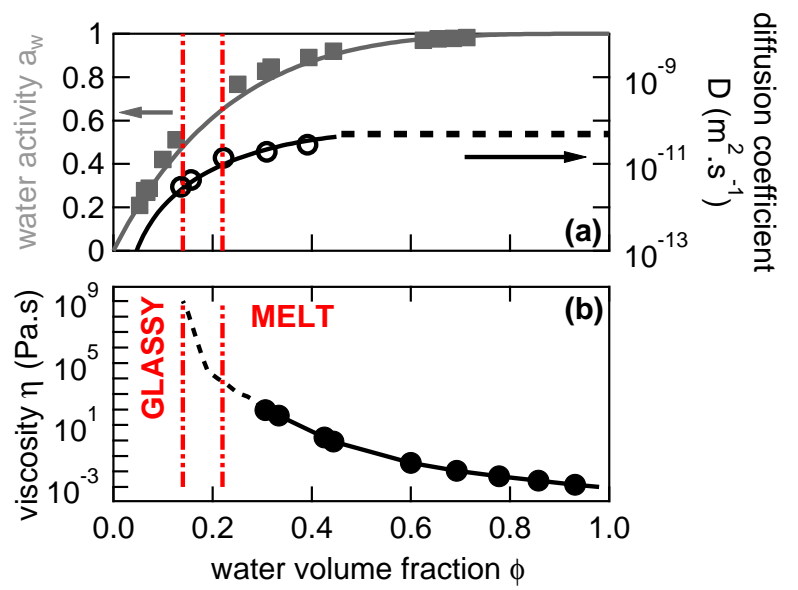

FIG. 1. (a) (left axis) Water activity $a_{w}$ in the atmosphere and (right axis) mutual diffusion coefficient versus volume fraction of water in the polymer at equilibrium. Dashed line: $D$ extrapolates to $D_{\text {self }}$ of the polymer for $\phi=1$. Sorption isotherm $a_{w}(\phi)$ (gray squares) is fit to a Flory equation with Flory parameter $\chi=0.5$ (grey line). (b) Viscosity of maltodextrin solutions as a function of measured water volume fraction (symbols).

The experiments were performed in a well controlled geometry: a $3 \mu \mathrm{L}$-droplet of solvent spontaneously spreads on a layer of polymer (maltodextrin, molecular mass 2500 g.mol ${ }^{-1}$ ). The polymer layers are formed by either spin-coating or dip-coating maltodextrin solutions on silicon wafers. The thickness $e$ of the resulting layer ranges from $100 \mathrm{~nm}$ to $3.6 \mathrm{~mm}$. In order to control the initial water volume fraction in the substrate $\phi_{0}$, the samples are equilibrated in a sealed chamber of controlled humidity and the experiments are performed in the same chamber. The contact line is monitored from the side and the top by two synchronized video cameras. The recorded views allow for the simultaneous determination of the contact angle of the spreading droplet $\theta$ and its instantaneous velocity $U$. We check that quasi-stationary conditions hold. For sub-micron thick polymer films, interferences build up between the light reflected at the polymer/air and polymer/silicon interfaces. The resulting hue on the color images, recorded by the top video camera, is a function of the product the local average refraction index and thickness of the polymer layer ne [7], a change of hue therefore indicates a local thickness change. The sorption isotherm of maltodextrin in water was measured by thermogravimetric analysis. Figure 1a shows the variation of water activity $a_{w}$ as a function of the water volume fraction in the polymer $\phi$. As for most pairs of polymer and solvent, the sorption isotherm follows a Flory equation [15] and is strongly non-linear: at low humidity the polymer is poorly hygroscopic, whereas it becomes hygroscopic at large humidities. The self-diffusion coefficient of water in maltodextrin $D_{\text {self }}$ at different water volume fractions $\phi$ was measured using a NMR technique [1618] (See the Supplemental Material [19]) . We further computed the mutual diffusion coefficient, which is the relevant quantity, using the Flory isotherm for $\phi<0.6$ : $D=D_{\text {self }} \frac{\partial \ln \left(a_{w}\right)}{\partial \ln (\phi)}$. As shown in Fig. 1a, the diffusion coefficient drops down by two orders of magnitude between $\phi=0.4$ and a few percents. Large variations are also observed for the viscosity of maltodextrin solutions (Fig. 1b) that were measured using a strain-controlled rheometer. Finally, the water volume fraction at glass transition $\phi_{g}$ was deduced from Differential Scanning Calorimetry (DSC) experiments made with samples of different initial water content. From the DSC and NMR data, the range of water content corresponding to glass transition spans $\phi_{g} \sim[0.14 ; 0.22]$, corresponding to $a_{w}^{g} \sim[0.5 ; 0.65]$ at room temperature. Less volatile solvents of maltodextrin were used in the spreading experiments: 1,3propanediol, DMSO, and 2,3butanediol of respective saturation concentrations $4.24,1.75$ and 1.18 g. $\mathrm{m}^{-3}$, whereas it is $23 \mathrm{~g} . \mathrm{m}^{-3}$ for water.

Figure 2a shows the contact angle vs. contact line velocity curves of a water droplet spreading on polymer layers with different initial water volume fractions $\phi_{0}$. On the initially melt layer $\left(\phi_{0}>\phi_{g}\right)$, the initial value of the spreading angle is larger than $30^{\circ}$, and both velocity and contact angle further decrease with time as already observed in previous works [7]. That decrease results from hydration of the layer during spreading by water evaporation from the droplet and its further condensation on the substrate. When the substrate is initially glassy $\left(\phi_{0}<\phi_{g}\right)$, a decrease of both $\theta$ and $U$ is also observed during spreading. However, at high velocities, the slope of the $\theta-U$ curve is smaller than the one on the melt layer, and at intermediate velocity values, the $\theta-U$ curve exhibits a kink that corresponds to an abrupt change of the spreading angle. This change of regime is observed with all the solvents tested provided the polymer is initially glassy and its thickness is in a range which is detailed later. The coordinates of the kink are denoted $U_{g}$ and $\theta_{g}$. We show in the following that the observed kink results from the transition from a glassy to a melt state undergone by the polymer layer in the vicinity of the contact line. We emphasize that although glass transition is characterized by rather smooth variations of the properties, the steep $\theta$ variations result from the simultaneous change of solvent sorption, solvent coefficient diffusion and mechanical properties of the substrate. Qualitatively, for $U>U_{g}$ the substrate is poorly solvated and behaves as a poorly solvoscopic rigid material, dissipation therefore mainly occurs in the spreading liquid; as the substrate gets solvated, it turns to a highly solvoscopic soft viscoelastic material that is deformed at the contact line for $U$ smaller than but close to $U_{g}$; dissipation then mostly 
(a)
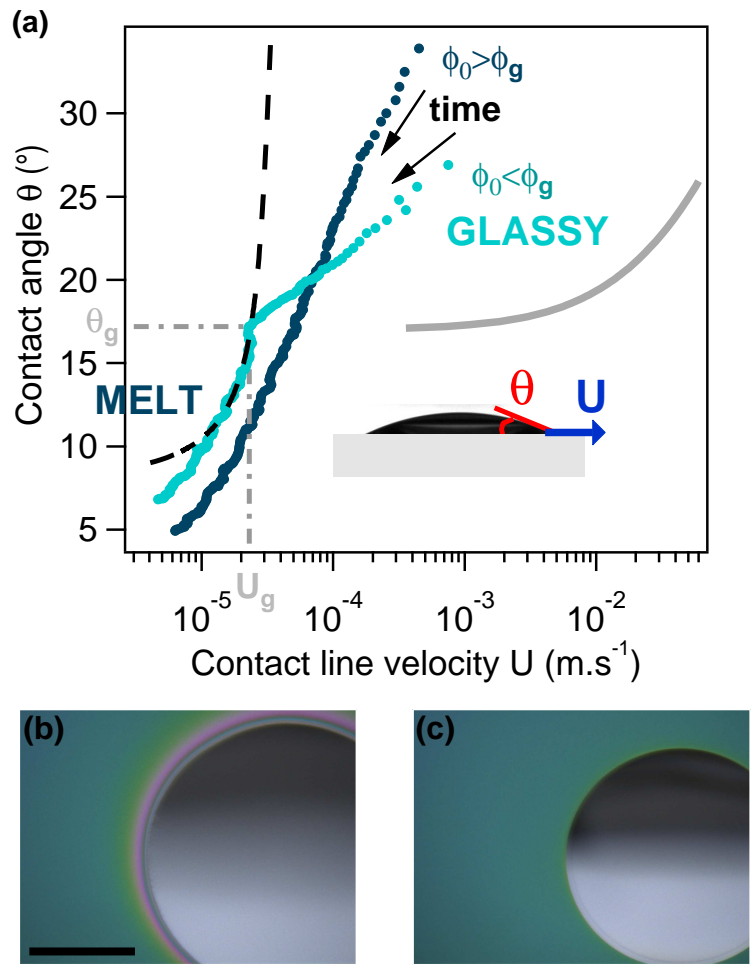

FIG. 2. (color online) (a) Contact angle as a function of contact line velocity of a water droplet spontaneously spreading on a $2.7 \mu \mathrm{m}$ thick layer of maltodextrin. The experimental data (dots) was obtained at two different initial humidities. The gray line represents Eq. 1 with $\theta_{e}=16^{\circ}, \gamma_{l}=0.07$ N.m ${ }^{-1}$, $\eta=10^{-3}$ Pa.s and $\ln (r)=10$ and the dashed black line Eq. 2 with $\theta_{e}=5^{o}, \gamma_{s}=0.1 \mathrm{~N} . \mathrm{m}^{-1}, m=0.75$ and $G_{0} \tau=2 \times 10^{3}$ Pa.s. Inset: side view of a spreading droplet. (b,c) Color images from the top of a droplet of DMSO spreading onto a 550-nm thick layer of maltodextrin. (b) $U<U_{g}$; (c) $U>U_{g}$. The scale bar represents $2 \mathrm{~mm}$.

occurs in the substate. The degree of hydration can be observed during spreading through the Newton hues that appear close to the droplet on the top views. Figure 2c indeed shows that the substrate is weakly swollen before glass transition, whereas it is significantly swollen over distances from the contact line close to $1 \mathrm{~mm}$ when in a melt state (Fig. 2b). As discussed later, the observed swelling results from the solvatation of the substrate, its deformation at the contact line occurring over distances too small to be visible on Figure $2 \mathrm{~b}$.

For a non soluble substrate, it has been shown that, if the substrate is rigid, dissipation occurs in the spreading liquid, and the wetting angle and contact line velocity are related through [20-22] :

$$
U=\frac{\theta \gamma_{l}\left(\cos \theta_{e}-\cos \theta\right)}{3 \eta_{l} \ln r}
$$

Where $\gamma_{l}$ is the liquid/air surface tension, $\theta_{e}$ the static wetting angle, $\eta_{l}$ the liquid viscosity, and $r$ the ratio of a macroscopic length scale (the droplet radius) and a microscopic cut off length $\kappa$, such that $\ln r \approx 10$ [13].

When the substrate is a soft viscoelastic material of elastic modulus $G^{\prime}$, it deforms at the contact line, owing to the vertical component of the capillary force $\gamma_{l} \sin \theta$ $[12,13]$. The vertical displacement is $\gamma_{l} \sin \theta / G^{\prime}$, and can reach several microns for a gel of elastic modulus $G^{\prime}=1 \mathrm{kPa}$. The deformed zone extends away from the contact line over a distance which is as well of a few microns, since it is given by the elastocapillary length $\gamma_{l} / G^{\prime}$. In that frame, the relation between wetting angle $\theta$ and contact line velocity $U$ on a non soluble soft substrate can be derived, provided a rheological model is chosen to describe the mechanical properties of the substrate. The Chasset-Thirion model used in ref. [13] is well suited to describe the behaviour of concentrated maltodextrin solutions [23]; the relaxation modulus writes $G(t)=G_{0}\left(1+\left(\frac{t}{\tau}\right)^{-m}\right)$, where $G_{0}$ is an elastic modulus, $\tau$ a relaxation time and $m$ an exponent. In that frame, a new $U$ vs. $\theta$ relation arises [13]:

$$
U=\left[\frac{\gamma_{s}\left(\cos \theta_{e}-\cos \theta\right)}{\gamma_{l} \sin ^{2} \theta}\right]^{1 / m} \frac{\gamma_{s}}{G_{0} \tau}
$$

where $\gamma_{s}$ is the air/substrate surface tension. Both equations 1 and 2 are represented in Fig. 2 for given values of the equilibrium angle $\theta_{e}$, surface tensions $\gamma_{s}$ and $\gamma_{l}$, and mechanical parameters $\tau, m$ and $G_{0}$. Although they are in qualitative agreement with the experimental data that exhibits larger slopes for $U<U_{g}$ than for $U>U_{g}$, those expressions do not provide a quantitative description for wetting on a soluble substrate. Indeed, in that case the volume fraction in the vicinity of the contact line varies with the velocity, but also with the distance to the contact line for a given velocity. This complexity prevents any further quantitative analysis. Equation 2 nevertheless provides an order of magnitude of the velocity $U_{g}$. Since the term to the power $1 / m$ is of the order of 1 , and since $\eta \approx G_{0} \tau$, equ. 2 actually yields $\eta U \approx \gamma_{s}$. For $\gamma_{s} \approx 0.1 N . m^{-1}$, the velocity $U_{g}=2.10^{-5} \mathrm{~m} . \mathrm{s}^{-1}$ measured in the experiment of Fig. 2 corresponds to $\eta \approx 5.10^{3}$ Pa.s, which is a good order of magnitude of the viscosity of maltodextrin with water volume fraction just above $\phi_{g}$.

As explained earlier, the solvent volume fraction close to the contact line mainly results from condensation of the solvent evaporated from the droplet. As a consequence, the solvent volume fraction in the vicinity of the contact line may not only depend on the velocity, but also on the ability of the polymer layer to uptake water, and thus on its thickness. The $\theta-U$ curves of Fig. 3 obtained with different substrate thicknesses exhibit a thickness dependence for thin enough substrates. For the two thickest layers and $U>U_{g}$, the contact angle is identical within the experimental uncertainty whereas it is strongly thickness-dependent in all the other cases. In- 


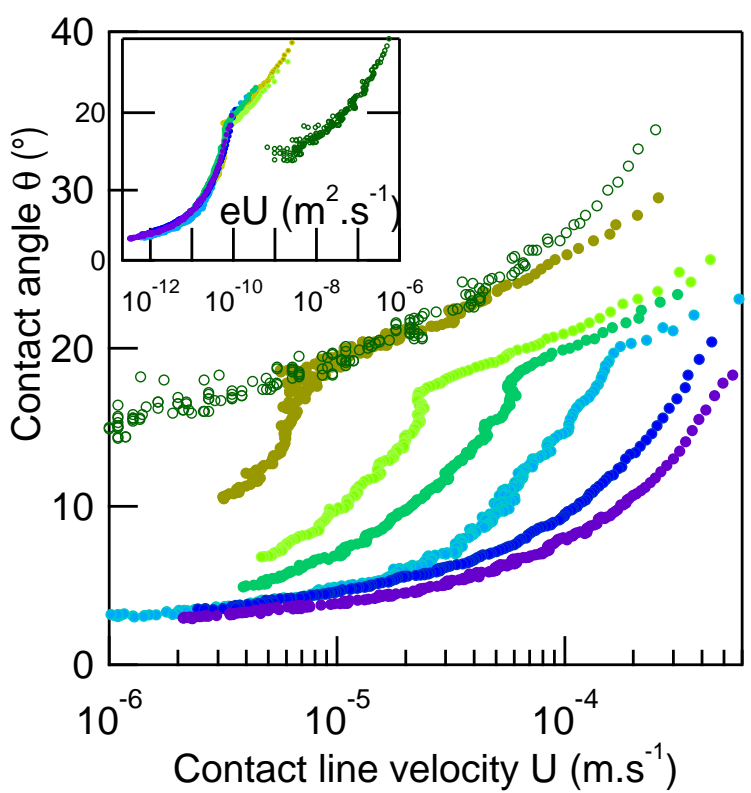

FIG. 3. Contact angle as a function of contact line velocity for water droplets spreading on maltodextrin of different thicknesses $e$. From top to bottom: $e=3.6 \mathrm{~mm}$ (open symbols), $8 \mu \mathrm{m}, 2.7 \mu \mathrm{m}, 1.1 \mu \mathrm{m}, 550 \mathrm{~nm}, 250 \mathrm{~nm}$ and $100 \mathrm{~nm}$. Insert: same data plotted vs. the product $e U$.

deed, the thinner the layer, the larger the solvent volume fraction is for a given solvent uptake. Consequently, the thinnest layers $(e=100 \mathrm{~nm}$ and $e=250 \mathrm{~nm})$ are solvated within a very short time and are observed to behave as melt layers although they are initially glassy. Oppositely, the thickest layer $(e=3.6 \mathrm{~mm})$ is never solvated enough during the experimental time to observe a change in wetting regime. Remarkably, for layers of intermediate thicknesses, the contact angle at which the wetting regime changes remains roughly constant at $\theta_{g} \sim 20 \pm 5$ for water droplets. That result is consistent with a change in wetting regime coinciding with glass transition: the wetting angle only depends on the water volume fraction in the substrate at the contact line, and thus $\theta_{g}=\theta\left(\phi_{g}\right)$. A simple scaling of the $\theta$ vs. $U$ variations when $U<U g$ can be inferred. At small velocity values and far enough from the contact line, solvent equilibrates along the film thickness within a time scale that is short compared to the other time scales. The substrate is then homogeneously solvated along the vertical direction. The distance from the contact line at which such a condition holds is given by [24] $: x_{c}=U^{2} e / D$. If $x_{c}$ is smaller than a microscopic cut off length $\kappa$, the problem can be described with a 1D model, the only spatial coordinate being the distance to the contact line. The water concentration then depends on the substrate thickness $e$ and velocity $U$ through the product $e U$ only. In that simple description, it is in particular possible to compute the water volume fraction in the substrate as it is reached by the contact line, $\phi_{c}$

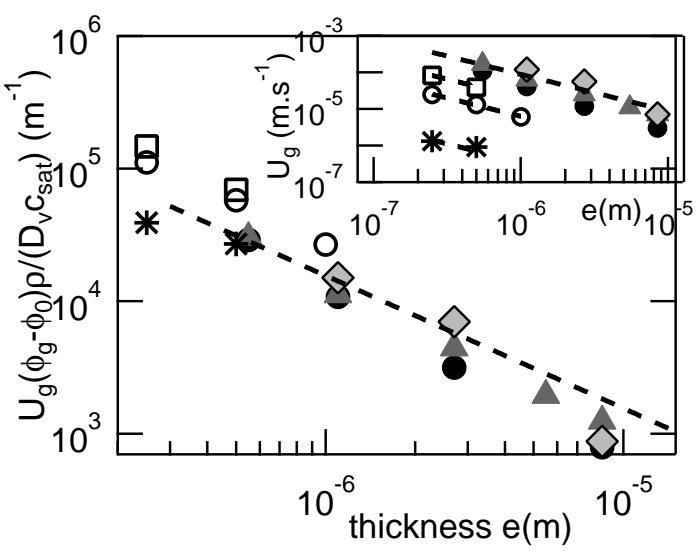

FIG. 4. $U_{g}\left(\phi_{g}-\phi_{0}\right) /\left(D_{v} c_{s a t}\right)$ as a function of polymer film thickness $e$ for water at water activities: 0.11 (circles), 0.43 (triangles); 0.58 (diamonds) and for solvents at $a_{w}=0$ (hollow circles: DMSO, stars: 2,3-butanediol, squares: 1,3propanediol). The dotted line represents $1 / e$ variations. Insert: velocity $U_{g}$ from the same experimental data. All data fit to $1 / e$.

$[9,24]:$

$$
\phi_{c}=\phi_{0}+\frac{1}{U e} \frac{D_{v} c_{s a t}}{\rho} \ln r
$$

Therefore, the wetting angle, which only depends on the water volume fraction $\phi_{c}$, is expected to be a function of $e U$ only. That result is well verified for all the data of Fig. 3 corresponding to velocities $U<U_{g}$ since they collapse on a master curve whatever the substrate thickness. Although the deviation from the master curve grows very slowly as the thickness increases, owing to the poor solvent sorption in that case, the scaling is not verified for $U>U_{g}$. Therefore, at $U=U_{g}$, the solvent has uniformly diffused over the substrate thickness at horizontal distances from the contact line larger than $\kappa$, and equ. 3 can be used to predict the value of $\phi_{c}$. Since velocity $U_{g}$ is actually reached when the water volume fraction at the contact line equals the water volume fraction at glass transition $\phi_{c}=\phi_{g}$, it yields for $U_{g}$ :

$$
U_{g}=\frac{1}{\phi_{g}-\phi_{0}} \frac{1}{e} \frac{D_{v} c_{s a t}}{\rho} \ln r
$$

That expression is compared to the experimental data in Fig. 4 where the measured values of $U_{g}$ are shown as a function of the substrate thickness $e$ for water droplets and different humidities. We find that $U_{g}$ varies as $1 / e$ in agreement with Eq.4. The expression for $U_{g}$ was also tested with other solvents of maltodextrin. Those solvents present similar values of diffusion coefficients in air $D_{v}$, and volume fractions at glass transition $\phi_{g}$ but very different saturation concentrations $c_{\text {sat }}$. Thus in equ. 4 , most of the $U_{g}$ solvent dependence, originates in the saturation concentration $c_{s a t}$ that 
strongly varies according to the solvent. As expected, we observe a good collapse on the same curve of all the values of the quantity $U_{g}\left(\phi_{g}-\phi_{0}\right) /\left(D_{v} c_{s a t}\right)$ for different solvents. We therefore conclude that the mechanism of solvent evaporation from the droplet and its further condensation in the substrate ahead the contact line governs whether the substrate experiences or not a glass transition, and that glass transition is responsible for deep changes in the wetting dynamics. In conclusion, we demonstrate that a glassy polymer substrate, when wetted by a polar solvent, may melt ahead the contact line, at contact line velocity smaller than a critical value, which depends on the layer thickness. Glass transition of the substrate results in a sharp decrease of the wetting angle, controlled by the substrate deformation induced by the contact line pulling.

[1] I. Schubert, in FOOD INGREDIENTS EUROPE, CONFERENCE PROCEEDINGS 1993 (Food Ingredients Europe, 1993) pp. 96-109, 1993 Food Ingredients Europe Conference, PORTE VERSAILLES, FRANCE, OCT 0406, 1993.

[2] P.-G. de Gennes, F. Brochard, and D. Quere, Gouttes, bulles, perles et ondes (Belin, 2002).

[3] D. Bonn, J. Eggers, J. Indekeu, J. Meunier, and E. Rolley, Reviews of Modern Physics 81, 739 (2009).

[4] J. T. Hirvi and T. A. Pakkanen, Journal of Chemical Physics 125 (2006), 10.1063/1.2356470.

[5] P. Muralidhar, E. Bonaccurso, G. K. Auernhammer, and H.-J. Butt, Coll. Polym. Sci 289, 1609 (2011).

[6] S. Farris, L. Introzzi, P. Biagioni, T. Holz, A. Schiraldi, and L. Piergiovanni, Langmuir 27, 7563 (2011).
[7] J. Dupas, E. Verneuil, M. Ramaioli, L. Forny, L. Talini, and F. Lequeux, Langmuir 29, 12572 (2013).

[8] C. Monteux, A. Tay, T. Narita, Y. De Wilde, and F. Lequeux, Soft Matter 5, 3713 (2009).

[9] J. Dupas, E. Verneuil, L. Talini, F. Lequeux, M. Ramaioli, and L. Forny, Interfacial Phenomena and Heat Transfer 1, 231 (2013).

[10] A. Tay, D. Bendejacq, C. Monteux, and F. Lequeux, Soft Matter 7, 6953 (2011).

[11] A. Marabi, G. Mayor, A. Raemy, I. Bauwens, J. Claude, A. S. Burbidge, R. Wallach, and I. S. Saguy, Food Research International 40, 1286 (2007).

[12] M. SHANAHAN and A. CARRE, Langmuir 11, 1396 (1995).

[13] D. Long, A. Ajdari, and L. Leibler, Langmuir 12, 5221 (1996).

[14] B. Zuo, C. Qian, D. Yan, Y. Liu, W. Liu, H. Fan, H. Tian, and X. Wang, Macromolecules 46, 1875 (2013).

[15] M. Rubinstein and R. Colby, Polymer Physics (University Press, Oxford, 2003).

[16] M. Van Landeghem, B. Bresson, B. Blümich, and J.B. d'Espinose de Lacaillerie, J. Magn. Reson. 211, 60 (2011).

[17] C. Johnson, Diffusion measurements by magnetic field gradient methods. In Encyclopedia of Nuclear Magnetic Resonance, edited by D. Grant and G. Harris (Wiley, New York, 1996).

[18] R. Kimmich, W. Unrath, G. Schnur, and E. Rommel, J. Magn. Reson. 91, 136 (1991).

[19] See Supplemental Material at [URL will be inserted by publisher] for the experimental procedure for measuring the self-diffusion coefficient of water in maltodextrin by NMR at different water volume fractions.

[20] O. V. Voinov, Fluid Dyn. 11, 714 (1976)

[21] R. G. Cox, J. Fluid Mech. 168, 169 (1986).

[22] P. G. de Gennes, Rev. Mod. Phys. 57, 827 (1985).

[23] C. Loret, V. Meunier, W. Frith, and P. Fryer, Carbohydrate Polymers 57, 153 (2004).

[24] A. Tay, C. Monteux, D. Bendejacq, and F. Lequeux, EPJ E 33, 8 (2010). 